\title{
Dural Arteriovenous Malformation in the Posterior Fossa - Case Report _-
}

\author{
MINORU SHIGEMORI*, MORIHISA SHIRAHAMA $†$, KUNITADA HARA $\dagger$, \\ TAKASHI TOKUTOMI $\dagger$ AND TOMOYUKI KAWABA*
}

\author{
Division of Neurosurgery, Omuta City Hospital, Omuta, 836 Japan
}

Received for publication March 4, 1981

\begin{abstract}
Summary: A rare case of dural arteriovenous malformation in the posterior fossa, successfully treated by craniotomy, is discussed. Clinical and angiographical characteristics, in addition to radical treatments for vascular anomalies, are.also described.
\end{abstract}

Key words: cerebro-vascular anomaly - dural arteriovenous malformation posterior fossa - meningeal artery - dural sinus

\section{Introduction}

Arteriovenous malformations (AVM's) which predominantly involve the intracranial dura mater are relatively rare. They have generally been termed dural arteriovenous malformations (Newton et al., 1968). The recent advent of angiographic techniques have allowed reliable identification of the definite vascular structures of these malformations. A successfully treated case of dural arteriovenous malformation in the posterior fossa is presented in this report.

\section{Case Report}

A 51-year-old female with hypertension was admitted to Omuta City Hospital on May 7, 1980, complaining of severe headache, vomiting and vertigo which developed abruptly two days prior to admission. On admission, she was alert and complained of vertigo and a severe headache in the occipital region. There was no history of head trauma. Neurological examination revealed neck stiffness and mild anisocoria, left pupil larger than right. There were no long-tract signs or cerebellar signs, however. Laboratory test revealed no abnormality. An emergency CT scan demonstrated a high density area over the cerebellar hemisphere indicating subarachnoid hemorrhage in the posterior fossa (Fig. 1). Nothing abnormal was found in the supratentorial area. Retrograde brachial angiogram on the left side showed a fine vascular anomaly at the upper surface of the right cerebellar hemisphere (Fig. $2 \mathrm{a}$, b). Right vertebral angiogram definitely identified vascular malformation at the right transverse sinus and torcular Herophili (Fig. $3 \mathrm{a}, \mathrm{b}$ ). The feeding arteries consisted of several meningeal branches of the right vertebral artery originating from the extracranial portion of the vertebral artery and superior hemispheric branch of

Present address: * Division of Neurosurgery, Shakaihoken Tagawa Hospital, Tagawa, 826

$\dagger$ Department of Neurosurgery, Kurume University School of Medicine, Kurume, 830 
the right superior cerebellar artery. A small aneurysmal dilatation of the distal branch of the right superior cerebellar artery was also observed. Right transverse sinus and distal portion of the superior sagittal sinus were well opacified at the arterial phase. These findings indicated dural arteriovenous malformation in the region of the torcular Herohili with reversal of blood flow into the sagittal sinus.

On June 19, 1980, following conservative treatment with antifibrinolytic agents and antihypertensive agents, a radical operation for the dural arteriovenous malformation was performed under suboccipital craniectomy. Prior to dural opening at the right posterior fossa, ablation of the periosteum from the occipital bone and coagulation of the meningeal branches identified preoperatively on the angiogram were carried out. The dura mater was then incised along the right transverse sinus and the occipital sinus, at which time the discoloration of both cerebellar surface caused by subarachnoid hemorrhage was noted. Three arterial branches from the right superior cerebellar artery supplying the dural AVM were successfully coagulated and an aneurysmal dilatation of the peripheral branch of the superior cerebellar artery on the right cerebellar surface was also ablated.

The patient made an uneventful recovery from the operation and was discharged on July 3, 1980. Histological examination of the aneurysmal dilatation showed a thrombosed vascular dilatation with anomalous vascular network, confirming arteriovenous malformation.

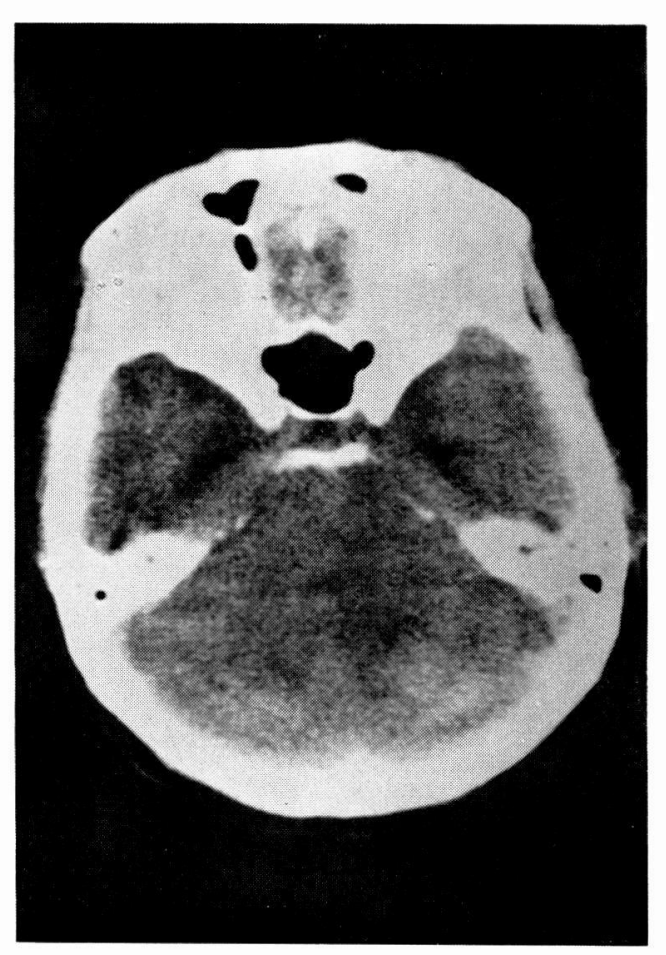

Fig. 1. CT scan on admission showes high density area over the cerebellar hemispheres indicating subarachnoid hemorrhage in the posterior fossa. 


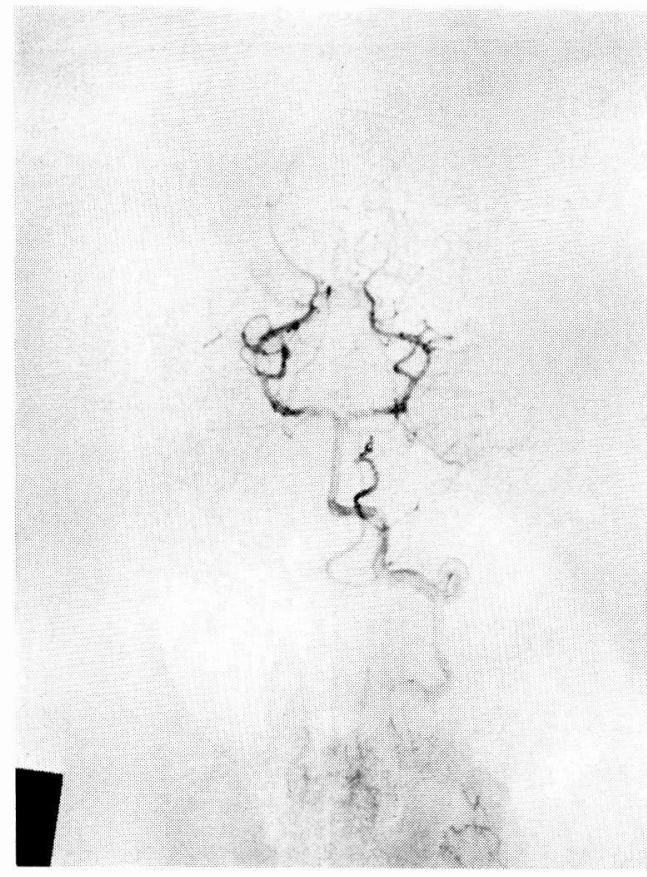

a. A-P view

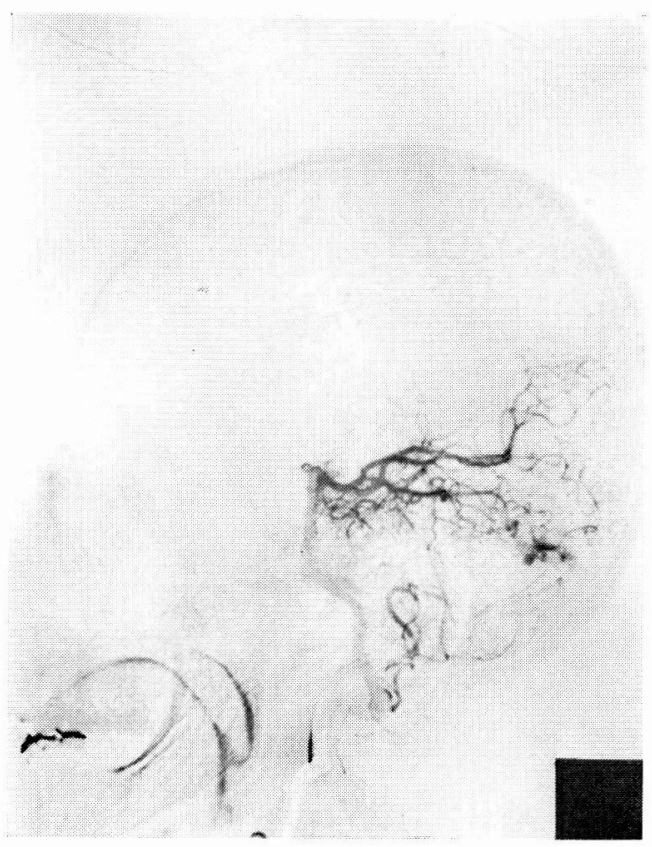

b. Lateral view

Fig. 2. Left vertebral angiogram showing a fine vascular abnormality at the upper surface of the right cerebellar hemisphere.

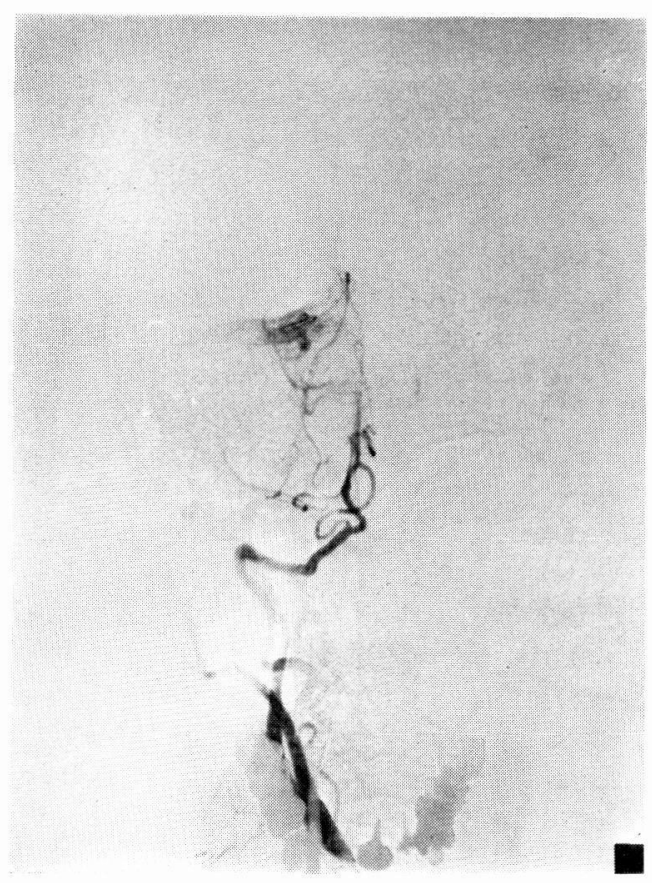

a. A-P view

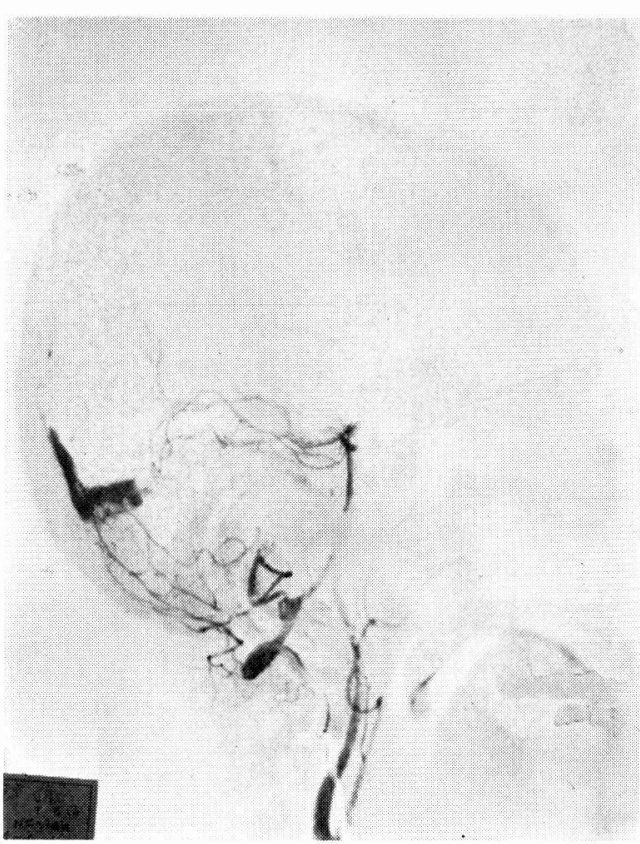

b. Lateralview

Fig. 3. Right vertebral angiogram showing dural arteriovenous malformation at the transverse sinus and torcular Herophili. The feeding arteries from the meningeal branches of the vertebral artery and from the superior cerebellar artery on the right side are also well demonstrated. 


\section{Discussion}

The congenital vascular malformations between the arterial branch and the basal emissary veins that drain the extracranial structures into the dural sinuses is generally termed dural arteriovenous malformation (Newton et al., 1968). These vascular malformations, however, have been recorded in the literature under different names: spontaneous carotid-cavernous fistula, dural vascular anomalies or malformations (Aminoff, 1973), arteriovenous fistula or arteriovenous aneurysm of the region of the transverse-sigmoid sinus (Obrador, 1975), and dural arteriovenous malformation in the posterior fossa (Newton et al, 1968). The meningeal branches of the internal and external carotid arteries and of the vertebral arteries are the major arterial components which communicate directly with the dural veins and sinuses (Aminoff, 1973) in these cases.

In 1969, Newton and Cronqvist reviewed the angiograms of 129 cases with intracranial AVM's and classified them on the basis of their arterial supply as pure pial, mixed pial and dural, and pure dural. They reported that $80 \%$ arose in the cerebral parenchyma. Pure dural AVM's were found in 15 cases, 6 supratentorial and 9 infratetorial. Mixed dural-pial AVM's such as seen in the present report, were found in 20 instances, 16 supratentorial and 4 infratentorial. Ohata and Kajikawa (1978) reviewed 164 cases with dural AVM's and found that $63 \%$ were in the transverse-sigmoid sinus, and $26 \%$ in the cavernous sinus.

The clinical characteristics of dural AVM include female predominance and diagnosis between age 40 and 60 years (Newton and Cronqvist, 1969; Aminoff, 1973; Ohata and Kajikawa, 1978). It is also well known that the signs and symptoms of dural AVM's may be indistinguishable from those of AVM's of the brain, although dural AVM's are extracerebral
(Kosnik et al., 1974). Either lesions may present with a bruit, subarachnoid hemorrhage, headache, hydrocephalus or neurological dificit (Kosnik et al, 1973; Obrador et al, 1675). According to Ohta and Kajikawa (1978), intracranial bruit (85\%), headache or ocular pain $(43 \%)$ and visual disturbance or papilledema (36\%) were the most common signs in patients with dural AVM's in the posterior fossa. Subarachnoid hemorrhage, as seen in the present case however is relatively uncommon (Newton et al., 1968). Secondary arterial and venous dilatation of the brain evoked by these AVM's has been suggested as a possible cause (Kosnik et al., 1974).

Based on the angiographic features of dural AVM's in the posterior fossa described in the literature (Newton et al., 1968; Ohata and Kajikawa, 1978), the most frequent arterial supplies are the occipital artery, hypophyseal trunk of the internal carotid artery, middle meningeal artery, and posterior meningeal artery from the vertebral artery. Blood supply from the superior cerebellar artery, as seen in the present case, was found in only one instance (Ohata and Kajikawa, 1978). The primary venous drainage was noted to be transverse-sigmoid sinus and other cortical veins or sinuses; retrograde drainage to the straight sinus was also frequently noted (Obrador et al, 1975, Ohta and Kajikawa, 1978).

The principle procedure in surgical treatment of dural AVM's is surgical occlusion or removal of the vascular malformation (Aminoff, 1973; Takatu and Suzuki, 1975). However, dural AVM's are often situated along the base of the skull and frequently drain directly into the dural sinus. Thus total removal of the lesion in the posterior fossa is not always possible. Many surgical approach have been suggested, including ligation of the external carotid artery, ablation of the periosteum from the occipital bone, occipital or/ and suboccipital craniectomy and clipping 
of the majority of feeding arteries at dura mater and tentorium, and shunting (Takatu and Suzuki, 1975). With these surgical procedures, coagulation and obliteration of all vascular channels and complete isolation of the sinuses may be obtained. Furthermore, as in this case, the dural AVM can be completely obliterated by a combination of methods.

\section{References}

Aminoff, M. J. (1973). Vascular anomallies in the intracranial dura mater. Brain, 96, 601612.

Kosnik, E. J., Hunt, W. E. and Miller, C. A. (1974). Dural arteriovenous malformations. J. Neurosurg. 40, 322-329.

Newton, T.H., Weidner, W. and Greitz. (1968).
Dural arteriovenous malformation in the posterior fossa. Radiology, 90, 27-35.

Newton, T. H. and Cronqvist, S. (1968). Involvement of dural arteries in intracranial arteriovenous malformations. Radiology, 93, 1071-1078.

Онта, T. and KajiKawa, H. (1978). Dural arteriovenous malformation. Neurol. Med. Chir. (Tokyo), 18, part 11, 439-472.

Obrador, S., Soto, M. and Silvela, J. (1975). Clinical syndromes of arteriovenous malformations of the transverse-sigmoid sinus. J. Neurol. Neurosurg. and Psychiat. 38, 436451.

TAKaku, A. and Suzuki, J. (1975). Dural arteriovenous malformation of the posterior fossa. - Clinical and angiographical analysis of 6 cases - Proceedings of the 4 th Conference of Surgical Treatment of stroke, 4, 8894. 